\title{
Study of the anomalous magnetic moment of the muon computed from the Adler function
}

\author{
Michele Della Morte ${ }^{1}$, Anthony Francis ${ }^{2}$, Gregorio Herdoiza ${ }^{4}$, Hanno Horch ${ }^{* 3}$, \\ Benjamin Jäger ${ }^{5}$, Andreas Jüttner ${ }^{6}$, Harvey Meyer $^{3}$, Hartmut Wittig ${ }^{2,3}$ \\ ${ }^{1}$ CP3-Origins \& Danish IAS, University of Southern Denmark \\ Campusvej 55, DK-5230 Odense M, Denmark and IFIC (CSIC) \\ Calle Catedrático José Beltrán, 2. E-46980, Paterna, Spain \\ ${ }^{2}$ Helmholtz Institute Mainz, Johannes Gutenberg Universität Mainz, 55099 Mainz, Germany \\ ${ }^{3}$ PRISMA Cluster of Excellence, Institut für Kernphysik, Johannes Gutenberg Universität Mainz, \\ 55099 Mainz, Germany \\ ${ }^{4}$ Instituto de Física Teórica UAM/CSIC and Departamento de Física Teórica, \\ Universidad Autónoma de Madrid, Cantoblanco, E-28049 Madrid, Spain \\ ${ }^{5}$ Department of Physics, College of Science, Swansea University, SA2 8PP, Swansea, UK \\ ${ }^{6}$ School of Physics and Astronomy \\ University of Southampton, UK \\ E-mail:horch@kph.uni-mainz.de, dellamor@ific.uv.es \\ francis@kph.uni-mainz.de, gregorio.herdoiza@uam.es, \\ B. Jaeger@swansea.ac.uk, a.juttner@soton.ac.uk, \\ meyerh@kph.uni-mainz.de, wittig@kph.uni-mainz.de
}

\begin{abstract}
We compute the Adler function on the lattice from vacuum polarization data with twisted boundary conditions using numerical derivatives. The study is based on CLS ensembles with two flavours of $O(a)$ improved Wilson fermions. We extrapolate the lattice data for the Adler function to the continuum limit and to the physical pion mass and analyze its dependence on the momentum transfer. We discuss the application of this method to the extraction of the $u, d$ contribution to $a_{\mu}^{\mathrm{HLO}}$.
\end{abstract}

The 32nd International Symposium on Lattice Field Theory

23-28 June, 2014

Columbia University New York, $N Y$

\footnotetext{
* Speaker.
} 


\section{Introduction}

The anomalous magnetic moment of the muon is given by $a_{\mu} \equiv\left(g_{\mu}-2\right) / 2$, where $g_{\mu}$ is the gyromagnetic factor of the muon. There has been a persistent deviation for the results obtained from theory and experiment [1] which is currently $\sim 3.6 \sigma$,

$$
\begin{aligned}
a_{\mu}^{e x p} & =116592091(54)(33) \cdot 10^{-11}, \\
a_{\mu}^{t h} & =116591803(01)(42)(26) \cdot 10^{-11} .
\end{aligned}
$$

This discrepancy may hint at new physical processes beyond the Standard Model. A new experiment at Fermilab aims at reducing the experimental error by a factor of four [2]. To fully exploit this level of accuracy, it is mandatory to also increase the theoretical precision. The error budget of the theoretical result is dominated by QCD effects. The error due to the leading order $\left(\alpha^{2}\right)$ contribution is $42 \cdot 10^{-11}$, and for the next to leading order $\left(\alpha^{3}\right)$ contribution it is $26 \cdot 10^{-11}$, while effects from weak interactions and QED are of the order of $10^{-11}$ and $10^{-13}$, respectively. Lattice QCD allows us to determine the leading hadronic contribution to the anomalous magnetic moment of the muon $\left(a_{\mu}^{\mathrm{HLO}}\right)$ from the hadronic vacuum polarization (VP). The VP tensor is given by

$$
\Pi_{\mu v}\left(Q^{2}\right)=\int d^{4} x e^{i Q x}\left\langle J_{\mu}(x) J_{v}(0)\right\rangle, \quad \Pi_{\mu v}\left(Q^{2}\right)=\left(Q_{\mu} Q_{v}-\delta_{\mu v} Q^{2}\right) \Pi\left(Q^{2}\right),
$$

where the second relation follows from Euclidean invariance and current conservation. In order to perform the convolution integral [3, 4], which yields $a_{\mu}^{\mathrm{HLO}}$, one needs the renormalized VP, $\hat{\Pi}\left(Q^{2}\right)=4 \pi^{2}\left(\Pi\left(Q^{2}\right)-\Pi(0)\right)$, i.e.

$$
\begin{aligned}
a_{\mu}^{\mathrm{HLO}} & =\left(\frac{\alpha}{\pi}\right)^{2} \int_{0}^{\infty} d Q^{2} f\left(Q^{2}\right) \hat{\Pi}\left(Q^{2}\right), \\
f\left(Q^{2}\right) & =\frac{m_{\mu}^{2} Q^{2} Z^{3}\left(1-Q^{2} Z\right)}{1+m_{\mu}^{2} Q^{2} Z^{2}}, \quad Z=-\frac{Q^{2}-\sqrt{Q^{4}+4 m_{\mu}^{2} Q^{2}}}{2 m_{\mu}^{2} Q^{2}},
\end{aligned}
$$

to obtain $a_{\mu}^{\mathrm{HLO}}$. This has been studied by a number of lattice collaborations [5-8]. This work is a continuation of [9], and is part of the $g-2$ project in Mainz, cf. [7, 10-12]. The largest contribution to the integral in eq. (1.2) is dominated by the low $Q^{2}$ region around $m_{\mu}^{2}$, which is also a difficult region to probe on the lattice, since statistical fluctuations rapidly increase as $Q^{2} \rightarrow 0$. While we cannot circumvent the need for precise data in this region, we can remove the need to extrapolate to $\Pi\left(Q^{2}=0\right)$. This can be achieved by studying the Adler function [13], defined as

$$
D\left(Q^{2}\right)=12 \pi^{2} Q^{2} \frac{d \Pi\left(Q^{2}\right)}{d\left(Q^{2}\right)} .
$$

Making use of partially twisted boundary conditions [14-16], we apply two procedures to determine the numerical derivative of $\Pi\left(Q^{2}\right)$ and to check for inherent systematic effects.

\section{Lattice setup and determination of the Adler function}

Our study is based on CLS [17 - 19] ensembles with $N_{f}=2 O(a)$-improved Wilson fermions, cf. table 1 , where partially twisted boundary conditions are imposed on the valence quarks. 


\begin{tabular}{|c|c|c|c|c|c|c|c|}
\hline Label & $\mathrm{V} / a^{4}$ & $\beta$ & $a[\mathrm{fm}]$ & $m_{\pi}[\mathrm{MeV}]$ & $m_{\pi} L$ & $N_{\text {cnfg }}$ & $N_{\text {meas }}$ \\
\hline A3 & $64 \times 32^{3}$ & 5.20 & 0.079 & 473 & 6.0 & 251 & 1004 \\
A4 & $64 \times 32^{3}$ & 5.20 & 0.079 & 363 & 4.7 & 400 & 1600 \\
A5 & $64 \times 32^{3}$ & 5.20 & 0.079 & 312 & 4.0 & 251 & 1004 \\
B6 & $96 \times 48^{3}$ & 5.20 & 0.079 & 267 & 5.1 & 306 & 1224 \\
\hline E5 & $64 \times 32^{3}$ & 5.30 & 0.063 & 456 & 4.7 & 1000 & 4000 \\
F6 & $96 \times 48^{3}$ & 5.30 & 0.063 & 325 & 5.0 & 300 & 1200 \\
F7 & $96 \times 48^{3}$ & 5.30 & 0.063 & 277 & 4.2 & 250 & 1000 \\
G8 & $128 \times 64^{3}$ & 5.30 & 0.063 & 193 & 4.0 & 205 & 820 \\
\hline N5 & $96 \times 48^{3}$ & 5.50 & 0.050 & 430 & 5.2 & 347 & 1392 \\
N6 & $96 \times 48^{3}$ & 5.50 & 0.050 & 340 & 4.1 & 559 & 2236 \\
O7 & $128 \times 64^{3}$ & 5.50 & 0.050 & 261 & 4.4 & 138 & 552 \\
\hline
\end{tabular}

Table 1: The CLS ensembles used in this study with the lattice spacing from [20] and the number of configurations $N_{\text {cnfg }}$ and measurements $N_{\text {meas }}$.

From eqs. (1.1) one can determine the VP function on the lattice. As an example we show the measurement for the ensemble F7 with $a=0.063 \mathrm{fm}$ and $m_{\pi}=277 \mathrm{MeV}$ in fig. 1 . To compute the Adler function we apply a linear interpolation within a certain interval of the data. The interpolation interval must be chosen with care: On the one hand, local fluctuations in the VP data may spoil a reliable determination of the numerical derivative if the interval is chosen too small. At the same time choosing too large a fit window cannot model the curvature accurately. We developed two procedures which use different criteria to select the best fit window at each $Q^{2}$ value.

The first procedure (proc. I) uses linear fits $\Pi_{f i t}\left(Q^{2}\right)=a_{I}+b_{I} Q^{2}$ with several fit windows $\varepsilon \in$ $[0.1,1.0] \mathrm{GeV}^{2}$ at each momentum transfer. We select the best fit $\Pi_{f i t}\left(Q^{2}\right)$ from the region where $b_{I}$ is stable with respect to the fit window size $\varepsilon$. The Adler function is then given by $b_{I} Q^{2}$. The second procedure (proc. II) also uses linear fits $\Pi_{f i t}^{(l)}\left(Q^{2}\right)=a_{I I}+b_{I I} \log \left(Q^{2}\right)$ and quadratic fits $\Pi_{\text {fit }}^{(q)}\left(Q^{2}\right)=a_{I I}^{\prime}+b_{I I}^{\prime} \log \left(Q^{2}\right)+c_{I I}^{\prime} \log \left(Q^{2}\right)^{2}$ with same fit windows $\varepsilon \in[0.1,1.0] \mathrm{GeV}^{2}$ as for proc. I at each momentum transfer. We apply cuts to the curvature $c_{I I}^{\prime}$, and to the $\chi^{2} /$ dof. By requiring $b_{I I} \simeq b_{I I}^{\prime}$ we choose our final results from the remaining set of fits. The factor $b_{I I}$ then determines the Adler function.

As a cross check we fit a Padé-[1,2] ansatz to the VP data and compute its derivative. The results for these procedures are shown on the right in fig. 1 . We find that the three discussed procedures match within errors across the considered region of $Q^{2}$.

\section{The Adler function in the continuum at the physical point}

In order to take the continuum limit and extrapolate the Adler function to the physical pion mass we perform combined fits of the type

$$
D_{i j k}\left(Q^{2}\right)=A_{[i j]}\left(Q^{2}\right)\left(1+B_{k}(a, Q)+C\left(m_{\pi}, Q^{2}\right)\right),
$$

where the indices $i, j, k$ are labels for the different fit ansätze that we considered. The factor $A$ models the $Q^{2}$ dependence, $B$ describes lattice artifacts, and $C$ the light quark mass dependence. 

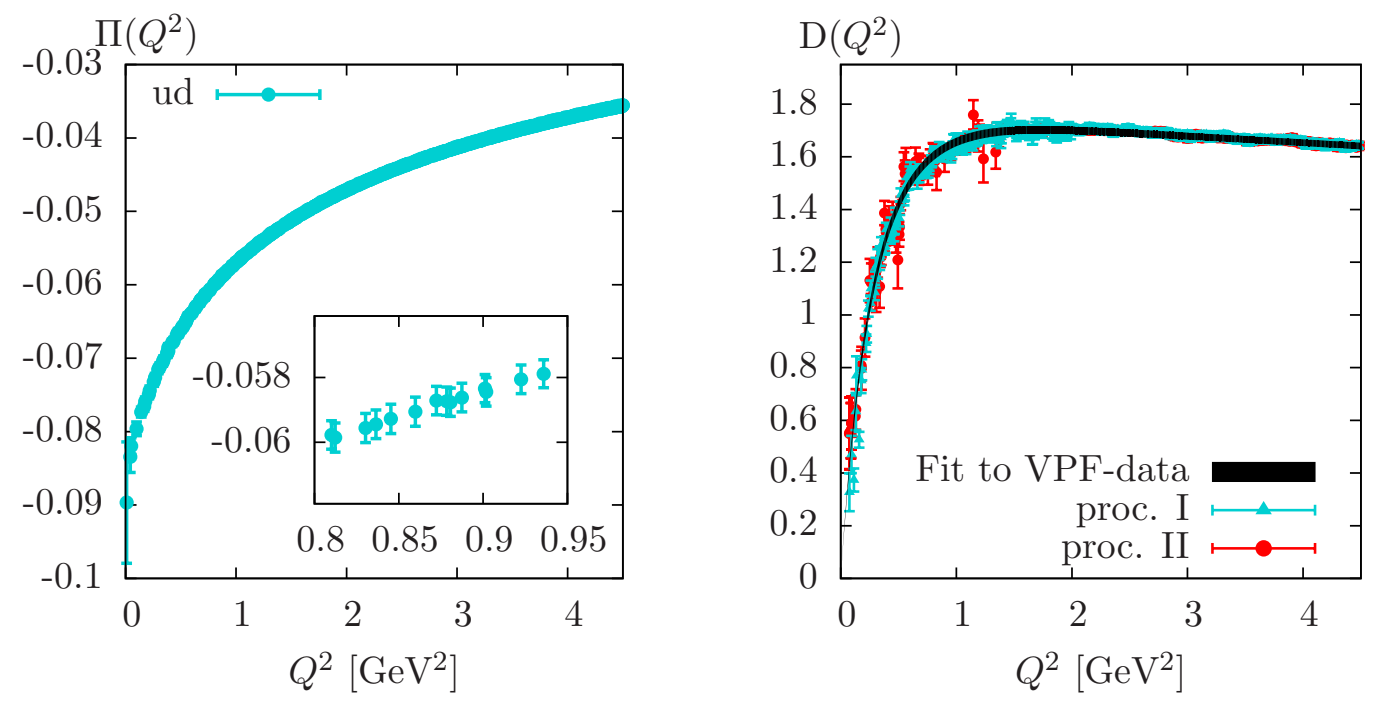

Figure 1: Left: The VP function on F7 with $a=0.063 \mathrm{fm}$ and $m_{\pi}=277 \mathrm{MeV}$. Right: Results for the Adler function using the two numerical procedures discussed in the text. The derivative of fitting the VP to a Padé[1,2]-Ansatz is shown as a cross check.

We use two ansätze for the momentum dependence

$A_{[12]}\left(Q^{2}\right)=Q^{2}\left(\frac{a_{1} b_{1}}{\left(b_{1}+Q^{2}\right)^{2}}+\frac{a_{2} b_{2}}{\left(b_{2}+Q^{2}\right)^{2}}\right), \quad A_{[22]}\left(Q^{2}\right)=Q^{2}\left(\frac{a_{1} b_{1}}{\left(b_{1}+Q^{2}\right)^{2}}+\frac{a_{2} b_{2}}{\left(b_{2}+Q^{2}\right)^{2}}+a_{0}\right)$,

which are based on derivatives of the Padé ansätze [1,2] and [2,2], cf. Refs. [7, 21]. To describe the lattice spacing dependence we consider two possibilities

$$
B_{1}(a, Q)=c_{1}(a Q)+c_{2}\left(4 \pi f_{\mathrm{K}} a\right), \quad B_{2}(a, Q)=c_{1}(a Q)^{2}+c_{2}\left(4 \pi f_{\mathrm{K}} a\right)^{2},
$$

where the term proportional to $c_{1}$ describes lattice artifacts which depend on the momentum transfer. The factor $4 \pi f_{K}$ in the momentum-independent term merely renders $c_{2}$ dimensionless. Furthermore, we explore two alternatives, namely that the leading lattice artifacts are either $\mathrm{O}(\mathrm{a})$ or $\mathrm{O}\left(\mathrm{a}^{2}\right)$. To describe the light quark mass dependence we use

$$
C\left(m_{\pi}, Q^{2}\right)=d_{1} \frac{m_{\pi}^{2}-\left(m_{\pi}^{\text {phys }}\right)^{2}}{d_{2}+Q^{2}} .
$$

In fig. 2 we show the result of an uncorrelated fit for the ansatz $D_{222}$ including all available ensembles, cf. table 1 , for the Adler function obtained using proc. II. The top left shows the Adler function data and the fit result as dotted, dashed, and solid lines for ensembles with different lattice spacings with a roughly constant pion mass of $m_{\pi} \approx 270 \mathrm{MeV}$. The continuous filled black curve is the extrapolation to the continuum at this pion mass. We find that lattice artifacts are small for the low $Q^{2}$ region, and grow for larger values of $Q^{2}$. This is further underlined by the plot on the top right where the slopes at $Q^{2}=1.0$ and $3.0 \mathrm{GeV}^{2}$ are compared.

The bottom left of fig. 2 shows the result for the Adler function data for a fixed lattice spacing and 

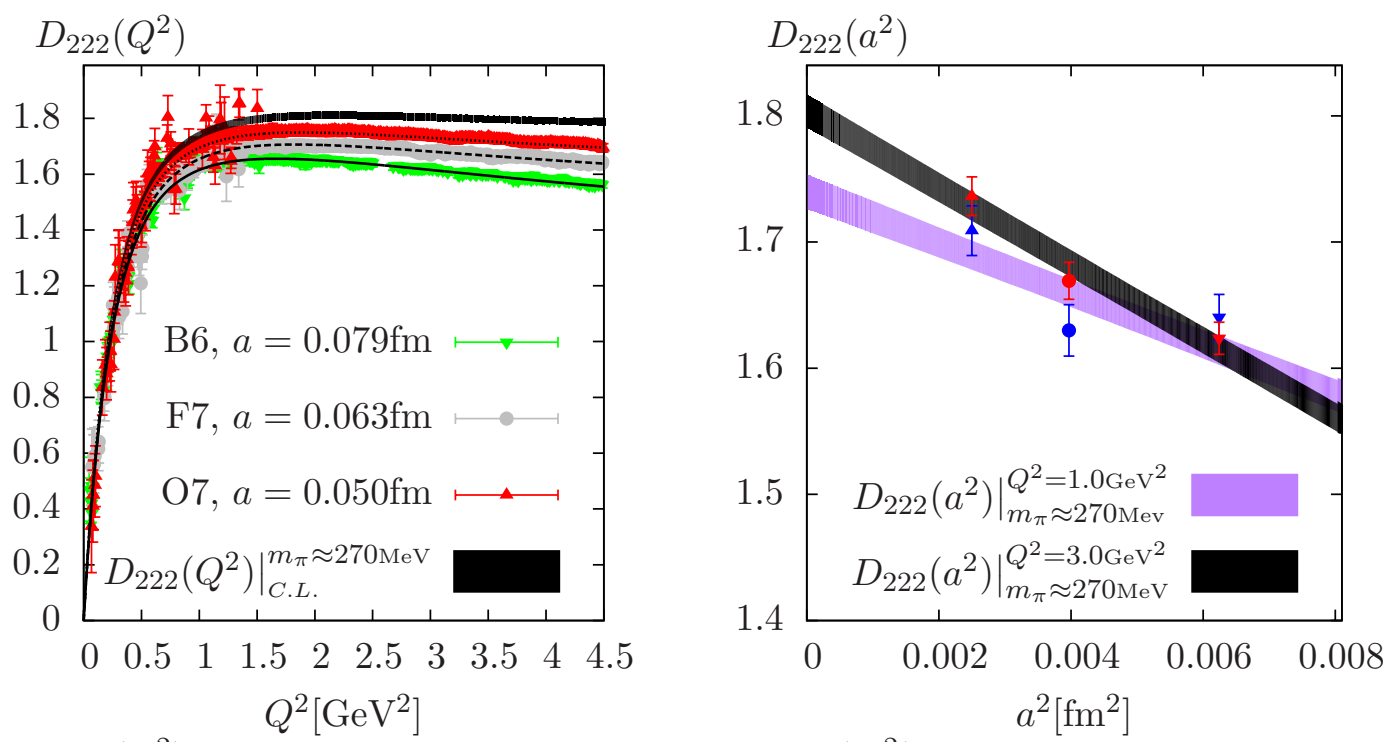

$D_{222}\left(Q^{2}\right)$
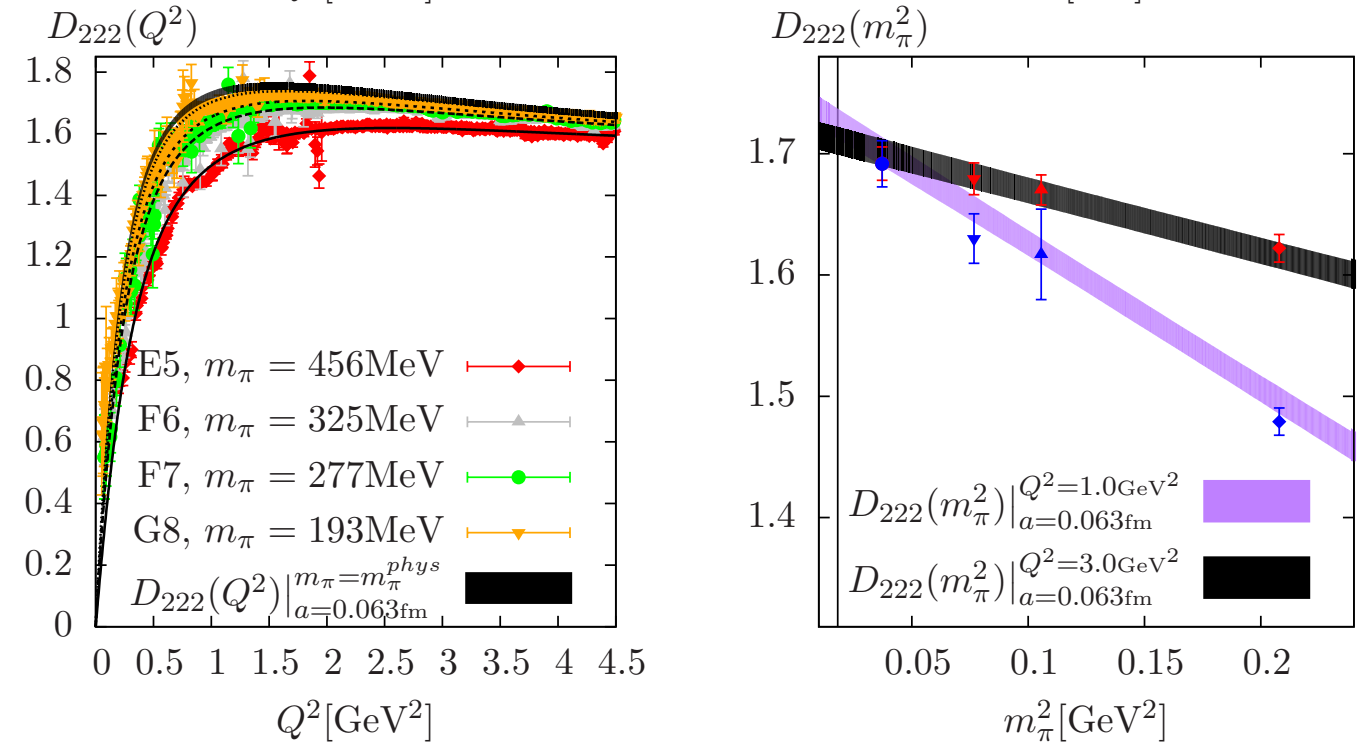

Figure 2: Illustration of an uncorrelated fit to the Adler function including the complete set of ensembles in table 1 for the Adler function obtained using proc. II with the ansatz $D_{222}$, i.e. a derivative of a Padé [2,2] ansatz with $O\left(a^{2}\right)$ effects. C.L. refers to the continuum limit. The results are discussed in the text. Top left: Comparison of the Adler function for the available lattice spacings and constant pion mass including the continuum extrapolation. Top right: Lattice spacing dependence at fixed $Q^{2}=1.0$ and $3.0 \mathrm{GeV}^{2}$. Bottom left: Comparison of the pion mass dependence fore a fixed lattice spacing including the extrapolation to the physical pion mass. Bottom right: Pion mass dependence at fixed $Q^{2}=1.0$ and $3.0 \mathrm{GeV}^{2}$.

the evaluation of the fit function for each pion mass shown as dashed, dotted, and solid lines. The continuous filled black curve represents the extrapolation to the physical pion mass. We observe that the pion mass dependence is mild at large $Q^{2}$ and becomes more and more significant when decreasing $Q^{2}$. The bottom right shows the dependence of the pion mass for two fixed momentum transfers. The vertical black line represents the physical pion mass. 


\section{Determination of $a_{\mu}^{\mathrm{HLO}}$}

$a_{\mu}^{\mathrm{HLO}}$ can be obtained via the integral $[22,23]$

$$
a_{\mu}^{\mathrm{HLO}}=\int_{0}^{1} d x g(x) D\left(\frac{x^{2} m_{\mu}^{2}}{1-x}\right), \quad g(x)=\frac{\alpha^{2}}{6 \pi^{2}} \frac{(1-x)(2-x)}{x}
$$

where we used the substitution $Q^{2} \rightarrow \frac{x^{2} m_{\mu}^{2}}{1-x}$ and inserted the Adler function $D\left(Q^{2}\right)$ in the continuum limit at the physical pion mass, obtained from the combined fit. The left of fig. 3 illustrates the integrand of eq. (4.1) for the case of the ensemble F7, $m_{\pi}=277 \mathrm{MeV}$ and $a=0.063 \mathrm{fm}$. The expected large contribution to $a_{\mu}^{\mathrm{HLO}}$ from momenta in the neighborhood of the muon mass (denoted by the vertical line in fig. 3) can be probed by the use of Padé approximants of different orders. The right of fig. 3 shows the results obtained for $a_{\mu}^{\mathrm{HLO}}$ using the different fit functions discussed in the previous section to the Adler function data obtained via proc. II. Lattice artifacts are well controlled, since similar results are obtained for $a_{\mu}^{\mathrm{HLO}}$ when using $O(a)$ and $O\left(a^{2}\right)$ ansätze. When all ensembles are included in the combined fit we observe a deviation for the two orders of the Padé ansätze that we considered. Removing the ensembles with $m_{\pi}>400 \mathrm{MeV}$ we find the deviation is reduced and the results agree within errors, which is in part due to an increase of the latter.
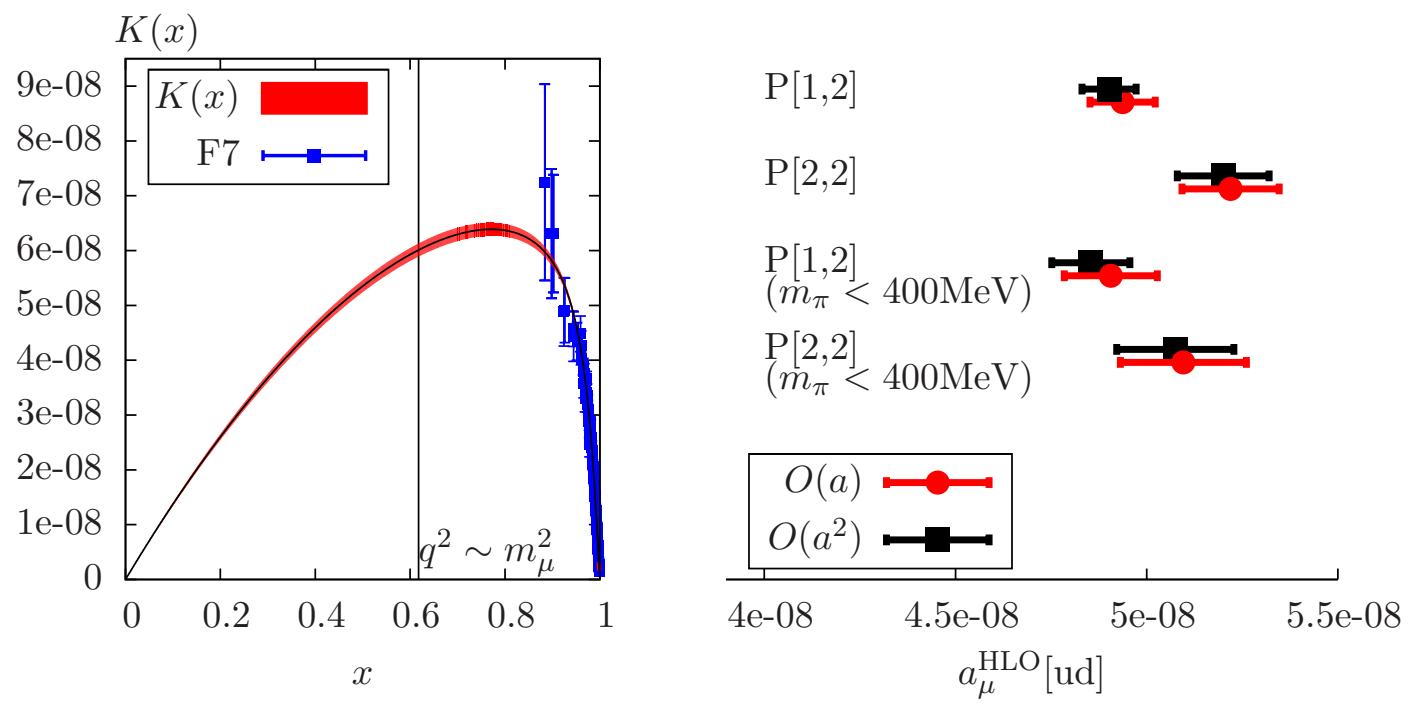

Figure 3: Left: Plot of the integrand of eq. (4.1) with $K(x)=g(x) D_{222}\left(\left(x^{2} m_{\mu}^{2}\right) /(1-x)\right)$ evaluated at the parameters of the $\mathrm{F} 7$ ensemble, in comparison with the data of the Adler function obtained via proc. II multiplied by $g(x)$. Right: Preliminary results of the $u, d$ contribution to $a_{\mu}^{\mathrm{HLO}}$ from uncorrelated fits to the Adler function data obtained via proc. II.

\section{Conclusions}

We presented two numerical procedures to determine the Adler function from $\Pi\left(Q^{2}\right)$ through lattice calculations. Both procedures match within errors for a large range in $Q^{2}$. After extrapolating the Adler function to the continuum and to the physical pion mass we use a kernel representation 
based on the Adler function to compute $a_{\mu}^{\mathrm{HLO}}$. We showed that lattice artifacts for $a_{\mu}^{\mathrm{HLO}}$ are small. We observe the results are sensitve to the chiral approach of the data to the physical point and the $Q^{2}$-dependence of the Padé approximants. We plan to investigate different ansätze to further study these effects. Furthermore, we will also extend this study to the strange and charm quark contribution to $a_{\mu}^{\mathrm{HLO}}$.

ACKNOWLEDGEMENTS: We thank Andreas Nyffeler for pointing out the kernel function in eq. (4.1) to us. Our calculations were performed on the "Wilson" and "Clover" HPC Clusters at the Institute for Nuclear Physics, University of Mainz. We thank Dalibor Djukanovic and Christian Seiwerth for technical support. This research has been supported in part by the DFG via the SFB 1044. G.H. acknowledges support by the the Spanish MINECO through the Ramón y Cajal Programme and through the project FPA2012-31686 and by the Centro de excelencia Severo Ochoa Program SEV-2012-0249. This work was granted access to the HPC resources of the Gauss Center for Supercomputing at Forschungzentrum Jülich, Germany, made available within the Distributed European Computing Initiative by the PRACE-2IP, receiving funding from the European Community's Seventh Framework Programme (FP7/2007-2013) under grant agreement RI-283493 (project PRA039) and ERC grant agreement No 279757.

\section{References}

[1] K. A. Olive et al. [Particle Data Group Collaboration], Chin. Phys. C 38 (2014) 090001.

[2] R. M. Carey, K. R. Lynch, J. P. Miller, B. L. Roberts, W. M. Morse, Y. K. Semertzides, V. P. Druzhinin and B. I. Khazin et al., FERMILAB-PROPOSAL-0989.

[3] E. de Rafael, Phys. Lett. B 322 (1994) 239, hep-ph/9311316.

[4] T. Blum, Phys. Rev. Lett. 91 (2003) 052001, hep-lat/0212018.

[5] C. Aubin and T. Blum, Phys. Rev. D 75 (2007) 114502, hep-lat/0608011.

[6] P. Boyle, L. Del Debbio, E. Kerrane and J. Zanotti, Phys. Rev. D 85 (2012) 074504, arXiv:1107.1497.

[7] M. Della Morte, B. Jäger, A. Jüttner and H. Wittig, JHEP 1203 (2012) 055, arXiv:1112.2894.

[8] F. Burger et al. [ETM Collaboration], JHEP 1402 (2014) 099, arXiv:1308.4327.

[9] H. Horch et al. PoS LATTICE 2013 (2013) 304, arXiv:1311.6975.

[10] G. Herdoíza, H. Horch, B. Jäger and H. Wittig, PoS LATTICE 2013 (2014) 444.

[11] A. Francis, B. Jäger, H. B. Meyer and H. Wittig, Phys. Rev. D 88 (2013) 054502, arXiv:1306.2532.

[12] A. Francis et al. arXiv:1410.7491.

[13] S. L. Adler, Phys. Rev. D 10 (1974) 3714.

[14] C. T. Sachrajda and G. Villadoro, Phys. Lett. B 609 (2005) 73, hep-lat/0411033.

[15] P. F. Bedaque and J. -W. Chen, Phys. Lett. B 616 (2005) 208, hep-lat/0412023.

[16] G. M. de Divitiis, R. Petronzio and N. Tantalo, Phys. Lett. B 595 (2004) 408, hep-lat/0405002.

[17] https://twiki.cern.ch/twiki/bin/view/CLS/WebIntro (2010).

[18] http://luscher.web.cern.ch/luscher/DD-HMC/index.html

[19] M. Marinkovic and S. Schaefer, PoS LATTICE2010 (2010) 031.

[20] S. Capitani et al. PoS LATTICE 2011 (2011) 145, arXiv:1110.6365.

[21] C. Aubin, T. Blum, M. Golterman and S. Peris, Phys. Rev. D 86 (2012) 054509, arXiv:1205.3695.

[22] B. e. Lautrup, A. Peterman and E. de Rafael, Phys. Rept. 3 (1972) 193.

[23] M. Knecht, Lect. Notes Phys. 629 (2004) 37, hep-ph/0307239. 